\title{
Fingering instability in buoyancy-driven fluid-filled cracks
}

\author{
T. TOUVET ${ }^{1} \uparrow$, N. J. B A L MFORTH $H^{2,3}$, R. V. CRASTER $R^{4,5} \ddagger$ \\ AND B. R. SUTHERLAN D \\ ${ }^{1}$ Département de Physique, École Normale Supérieure de Lyon, Université de Lyon, 46 allée d'Italie, \\ 69364 Lyon CEDEX 07, France \\ ${ }^{2}$ Department of Mathematics, University of British Columbia, 1984 Mathematics Road, \\ Vancouver, BC V6T 1Z2, Canada \\ ${ }^{3}$ Department of Earth and Ocean Science, University of British Columbia, 6339 Stores Road, \\ Vancouver, BC V6T 1Z4, Canada \\ ${ }^{4}$ Department of Mathematical and Statistical Sciences, University of Alberta, \\ Edmonton, T6G 2G1, Canada \\ ${ }^{5}$ Department of Mathematics, Imperial College London, South Kensington, London SW7 2AZ, UK \\ ${ }^{6}$ Department of Physics, University of Alberta, Edmonton, T6G 2G1, Canada \\ (Received 26 May 2010; revised 2 September 2010; accepted 9 November 2010; \\ first published online 24 February 2011)
}

The stability of buoyancy-driven propagation of a fluid-filled crack through an elastic solid is studied using a combination of theory and experiments. For the theory, the lubrication approximation is introduced for fluid flow, and the surrounding solid is described by linear elasticity. Solutions are then constructed for a planar fluid front driven by either constant flux or constant volume propagating down a pre-cut conduit. As the thickness of the pre-cut conduit approaches zero, it is shown how these fronts converge to zero-toughness fracture solutions with a genuine crack tip. The linear stability of the planar solutions towards transverse, finger-like perturbations is then examined. Instabilities are detected that are analogous to those operating in the surface-tension-driven fingering of advancing fluid contact lines. Experiments are conducted using a block of gelatin for the solid and golden syrup for the fluid. Again, planar cracks initiated by emplacing the syrup above a shallow cut on the surface of the gelatin develop transverse, finger-like structures as they descend. Potential geological applications are discussed.

Key words: fingering instability, magma and lava flow, thin films

\section{Introduction}

The propagation of fluid-filled cracks through elastic solids is of recurrent interest in several areas of geophysics, including the rise of magma through the lithosphere (Weertman 1971; Spence \& Turcotte 1990; Rubin 1995; Taisne \& Jaupart 2009), the migration of fluid-filled glacial crevasses (Weertman 1971), the detachment of glaciers from their bed (Tsai \& Rice 2010), the calving of icebergs (Weiss 2004) and the

$\dagger$ Present address: Département de Mécanique, École Polytechnique, 91128 Palaiseau CEDEX, France.

$\ddagger$ Email address for correspondence: r.craster@imperial.ac.uk or theo.touvet@ens-lyon.org 
drainage of supraglacial lakes (Krawczynski et al. 2009). In geological engineering, the use of hydraulic fracturing techniques is commonplace in enhancing the recovery of oil and gas (Desroches et al. 1994; Economides \& Nolte 2000; Adachi et al. 2007), and in carbon sequestration (Boschi et al. 2009).

The theory of buoyancy-driven cracks was pioneered by Spence and co-workers (Spence, Sharp \& Turcotte 1987) and Lister (Lister 1990a,b). These authors built solutions for the propagation of two-dimensional cracks or dykes. Notably, the crack profiles develop distinct bulges near their tips where the elastic restoring forces in the ambient solid restrain fluid flow. The profiles of the resulting planar cracks therefore have shapes that are reminiscent of fluid films with contact lines advancing down inclined planes, which develop pronounced ridges due to surface tension. The aim of the present work is to explore this analogy a little further and, in particular, determine whether planar buoyancy-driven cracks are prone to three-dimensional fingering instabilities, as found for contact lines (Huppert 1982; Silvi \& Dussan V. 1985; Oron, Davis \& Bankoff 1997; Craster \& Matar 2009). If so, there could well be important implications in the various practical applications. For example, fingering provides a mechanism for flow localization in planar magma dykes that is independent of thermal and solidification effects, which might explain geological observations such as regularly spaced volcanic features above subduction zones (Bloomer, Stern $\&$ Smoot 1989).

We approach the problem by first performing a theoretical analysis that follows along similar lines to that used for fluid contact lines (Troian et al. 1989; Spaid \& Homsy 1996). More specifically, we build planar crack solutions similar to those constructed by Spence, Lister and co-workers, and then test the linear stability of these solutions towards non-planar (transverse) perturbations. In order to accomplish the task, we make some simplifications and avoid some of the issues regarding the actual crack tip by assuming that the fluid flows up an existing, but relatively narrow conduit. This latter approximation is often used to regularize the singular behaviour of fluid contact lines; as we show here, this approximation is less significant for the crack problem because in the limit in which the thickness of the pre-existing conduit approaches zero, the solutions converge to that for a finite crack without fracture toughness (which has geophysical relevance, if not industrial).

Having established that fingering may occur from a theoretical perspective, we then move on to describe some experiments that demonstrate that the phenomenon can also be reproduced in the laboratory. Given the inaccessibility of the geophysical and petroleum engineering applications (often kilometres deep within the Earth and involving fluids at extreme pressures and temperatures), analogue laboratory experiments have been conducted previously to study buoyancy-driven cracks. In particular, experiments have been conducted on the migration of cracks through blocks of gelatin driven by negatively buoyant viscous fluids (Takada 1990; Heimpel \& Olson 1994; Menand \& Tait 2001, 2002; Taisne \& Tait 2009). None of these previous experimental studies have set up the geometrical arrangement in a way that allows for the possibility that an initially largely planar crack could finger in the transverse direction, which motivates our present experiments. The work by Taisne \& Tait (2009) is perhaps closest to our current attempts: their single three-dimensional crack is effectively one of our fingers. To whet the reader's appetite, we show in figure 1 a photograph of a fingering crack in a typical experiment. Note the build-up of fluid, or bulging of the conduit, near the advancing crack front (as evidenced by the darker shading of those regions, which reflects a thicker layer of the dyed fluid). 


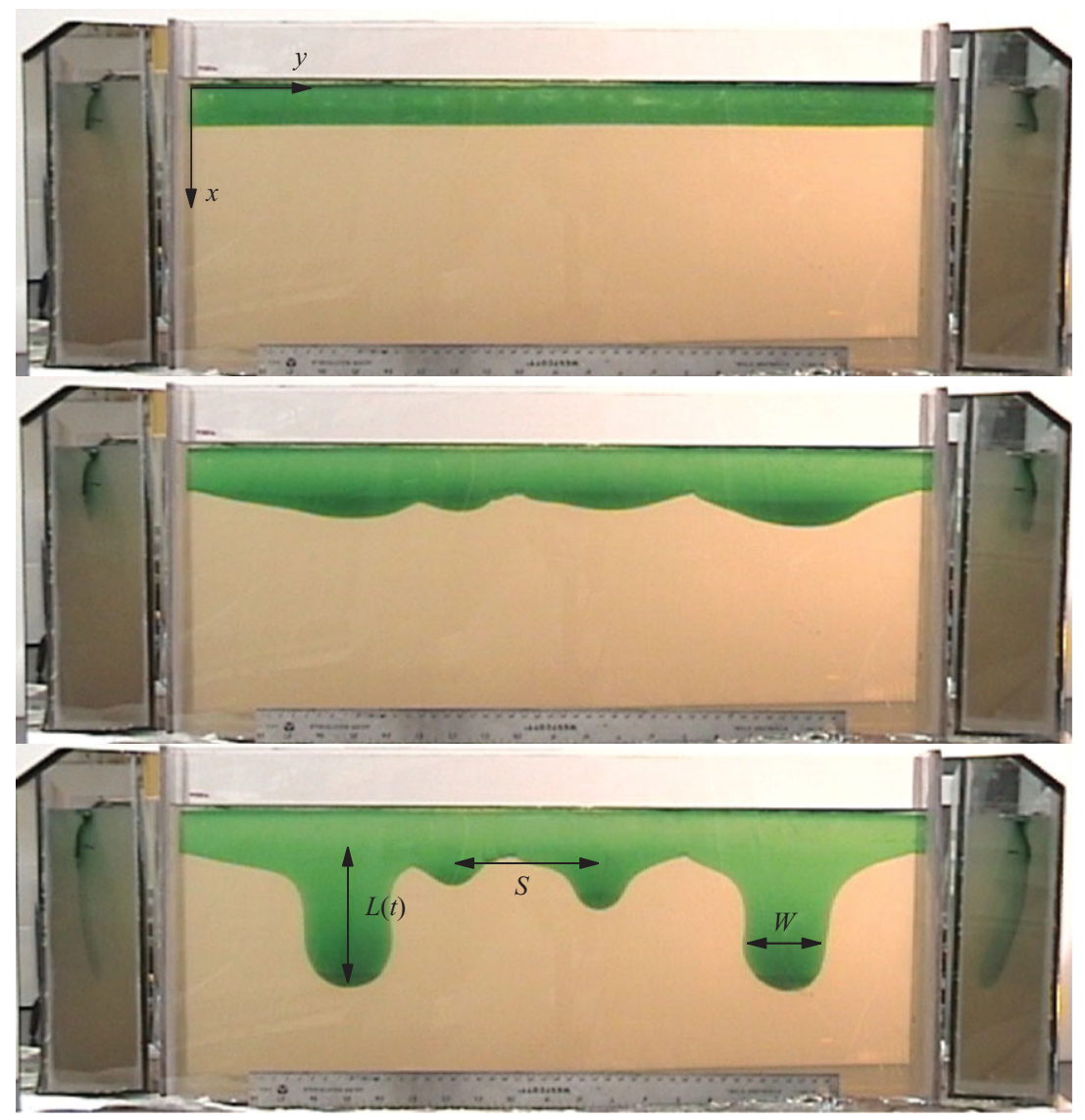

FIGURE 1. (Colour online) Three photographs showing a buoyancy-driven fluid-filled crack propagating through gelatin at times $t=0,400$ and $980 \mathrm{~s}$. The experiment is initiated by cutting a $4 \mathrm{~cm}$ deep slot filled with $45.36 \mathrm{~g}$ of Golden Syrup. Details of the experimental set-up and materials are provided in $\S 3$. The internal dimensions of the box are $59.6 \times 29.7 \times 7.2 \mathrm{~cm}$. Inclined mirrors at the ends of the tank display the crack cross-section. Diagnostic finger measurements are $L(t)$, the length of each finger, the maximal width of a well-developed finger $W$, and the spacing between those fingers $S$.

\section{Theoretical analysis}

Following previous authors (Spence et al. 1987; Lister 1990a), we assume that a long, thin fluid-filled crack lies within a homogeneous, isotropic elastic medium. The fluid density $\rho_{f}$ differs from the solid density $\rho_{s}$, resulting in a buoyancy force acting in the positive $x$-direction. The $z$-axis is orientated in the direction of the relatively narrow dimension of the crack, with $y$ pointing along the fissure, see figure 1 . The half-thickness of the crack is given by $z=h(x, y, t)$. The elastic solid is characterized by Young's modulus $E$ and Poisson ratio $v$, and we assume that the deformations are sufficiently small that linear theory applies. The fluid is isothermal and has viscosity $\mu$. We assume that the resulting fluid flow is sufficiently slow that the Reynolds number is small, and we may therefore apply Reynolds' lubrication approximation. 


\subsection{The equations}

Reynolds' lubrication equation is

$$
\frac{\partial h}{\partial t}=\nabla \cdot\left[\frac{h^{3}}{3 \mu} \nabla p\right]-\frac{\beta}{3 \mu} \frac{\partial h^{3}}{\partial x},
$$

where $p(x, y, t)$ is the fluid pressure (which is independent of $z$ to leading order) and $\beta=\left(\rho_{s}-\rho_{f}\right) g$ is the 'buoyancy'. Using Kelvin's fundamental solution for a slender planar crack opening under symmetrical compressional loading, $p$ is related to the opening displacement $2 h$ via the non-local relationship,

$$
\iint_{\Omega} \frac{h\left(x^{\prime}, y^{\prime}, t\right)}{R^{3}} \mathrm{~d} x^{\prime} \mathrm{d} y^{\prime}=-\frac{2 \pi(1-v)}{G} p(x, y, t),
$$

where the bulk modulus $G=E /[2(1+v)]$,

$$
R=\sqrt{\left(x-x^{\prime}\right)^{2}+\left(y-y^{\prime}\right)^{2}},
$$

and the integral is to be interpreted in the sense of the Hadamard finite part (e.g. Ioakimidis 1982). For a finite crack with an edge given by a genuine fracture, the domain of integration $\Omega$ denotes the projection onto the $(x, y)$-plane of the footprint of the crack, and the fracture position must be calculated as part of the solution of the resulting free-boundary problem. As mentioned in the introduction, however, although we consider buoyantly driven cracks, our approach to the problem is indirect: we do not introduce a finite edge to the crack and impose a fracture condition there, but instead pre-cut the solid with an infinitely long, planar conduit. Thus, for the present case, $\Omega$ is the entire $(x, y)$-plane.

We non-dimensionalize the equations using a typical crack half-width $\mathscr{H}$ for lengths in the $z$-direction, and a length scale based on a balance between elastic pressure and buoyancy $\mathscr{L}$ given by

$$
\mathscr{L}^{2}=\frac{G \mathscr{H}}{(1-v) \beta}
$$

for lengths in the crack plane (the $x$ - and $y$-directions). That is,

$$
h=\mathscr{H} \hat{h}, \quad x=\mathscr{L} \hat{x}, \quad y=\mathscr{L} \hat{y} .
$$

We scale the pressure and time according to

$$
p=\beta \mathscr{L} \hat{p}, \quad t=\frac{3 \mu \mathscr{L}}{\beta \mathscr{H}^{2}} \hat{t} .
$$

The hatted variables are all dimensionless, and since we use only these variables hereon we now discard the hat decoration. The dimensionless governing equations then read

$$
\frac{\partial h}{\partial t}=\nabla \cdot\left(h^{3} \nabla p\right)-\left(h^{3}\right)_{x}
$$

and

$$
\iint_{\Omega} \frac{h\left(x^{\prime}, y^{\prime}, t\right)}{R^{3}} \mathrm{~d} x^{\prime} \mathrm{d} y^{\prime}=-2 \pi p(x, y, t) .
$$

We consider two different configurations for advance of fluid down the pre-cut fissure, each of which dictates the boundary conditions. First, we consider a constant flux situation in which fluid is constantly fed in from $x \rightarrow-\infty$ with a flux $Q$ pushing open the conduit there to a given thickness. Taking the associated half-thickness to 
be the characteristic scale, $\mathscr{H}$, implies that $h \rightarrow 1$ as $x \rightarrow-\infty$, with $\mathscr{H}=(3 \mu Q / 2 \beta)^{1 / 3}$. The influx of fluid feeds a buoyant current that gradually opens up the conduit further along (provided $h(x, y, 0)$ is less than $\mathscr{H}$ ), but well ahead of that current, the half-thickness is given by that of the pre-cut conduit. Thus, denoting $b$ as the ratio of the half-thickness of the pre-cut conduit to $\mathscr{H}$, we impose $h \rightarrow b$ as $x \rightarrow \infty$. A convenient state from which to commence a planar initial-value problem is then

$$
h(x, y, 0)=\frac{1}{2}(1+b)-\frac{1}{2}(1-b) \tanh x .
$$

Provided $b$ is taken sufficiently small, this step-like initial condition is expected to steepen up into a propagating fluid front. Indeed, as shown below, those propagating fronts converge to a limiting solution as $b \rightarrow 0$ (unlike in the corresponding contactline problem, see Spaid \& Homsy 1996), which corresponds to a constant-flux buoyancy-driven crack propagating through an elastic solid with vanishing fracture toughness.

Second, we study a constant-volume problem in which a section of the pre-cut conduit is initially inflated with an additional volume of fluid. We take the maximum half-thickness of the inflated section to be $\mathscr{H}$, and then impose $h \rightarrow b$ as $x \rightarrow \pm \infty$. A planar initial condition suitable for this situation is then

$$
h(x, y, 0)=b+\frac{1}{2}(1-b)\{\tanh [\sigma(x+1)]-\tanh [\sigma(x-1)]\},
$$

and we choose $\sigma=20$ to get a smoothed 'top-hat' initial condition. The additional volume added to inflate the conduit is then of the order of $V_{0}=4 \mathscr{H} \mathscr{L}^{2}$, if $b \ll 1$, implying $\mathscr{H}=V_{0} / 4 \mathscr{L}^{2}$.

Although it is not necessary to incorporate fracture toughness into our problem, it remains helpful to estimate the magnitude of its effect in the experiment and geological applications. The relevant dimensionless measure (Roper \& Lister 2007) is

$$
\mathscr{K}=\frac{(1-v) K}{G \mathscr{H}}\left(\frac{2 \mathscr{L}}{\pi}\right)^{1 / 2},
$$

where $K$ is the fracture toughness (with units $\mathrm{Pa} \mathrm{m}^{1 / 2}$; Freund $1990 a$ ). As noted by Roper \& Lister (2007), the geological applications are characterized by $\mathscr{K} \ll 1$. In experimental situations $\mathscr{K}$ can be larger and we return to this issue in the discussion of $\S 4$.

\subsection{Constant-flux fronts}

Two-dimensional (planar) solutions, $h(x, y, t)=H(x, t)$ and $p(x, y, t)=P(x, t)$, to the initial-value problem with (2.9) satisfy

$$
H_{t}=\partial_{x}\left[H^{3}\left(P_{x}-1\right)\right]
$$

and

$$
P(x, t)=\frac{1}{\pi} f_{-\infty}^{+\infty} \frac{\partial H(z, t)}{\partial z} \frac{\mathrm{d} z}{(x-z)},
$$

subject to $H \rightarrow 1$ as $x \rightarrow-\infty$ and $H \rightarrow b$ as $x \rightarrow+\infty$; the decoration on the integral reminds us that the Cauchy principal value must be taken. As illustrated in figure 2, numerical solutions to the initial-value problem show rapid convergence to steadily propagating fronts. (For the computations, we approximate spatial derivatives with centred finite differences on a uniform grid of 2001 points and evaluate the integral using a simple trapezoid rule, then time step the resulting ordinary differential equations with a standard stiff integrator.) 


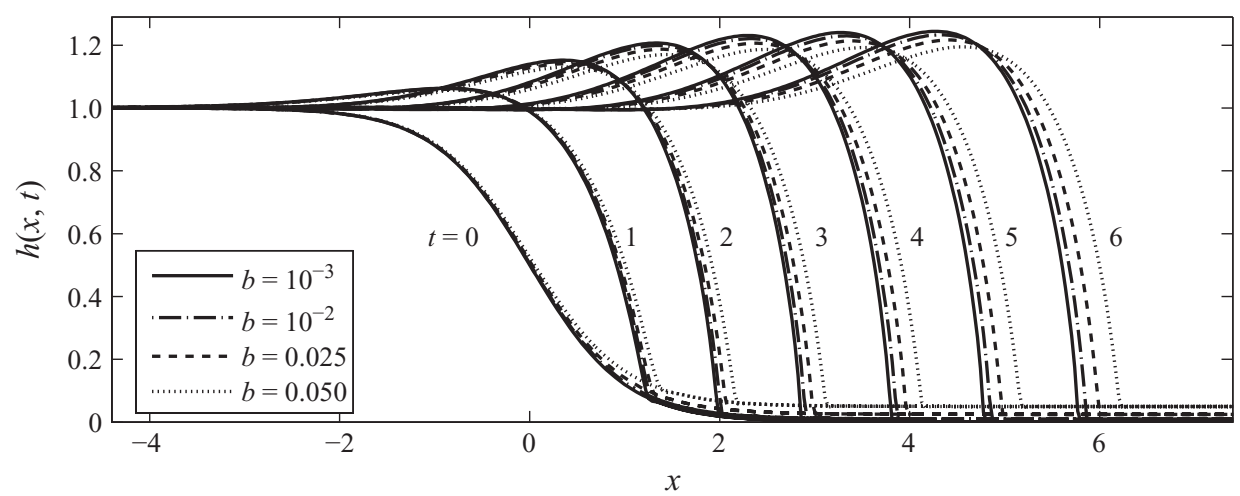

FIGURE 2. Snapshots of solutions to the two-dimensional initial-value problem with (2.9) for $b=0.05,0.025,10^{-2}$ and $10^{-3}$, at times $t=0,1, \ldots, 6$.

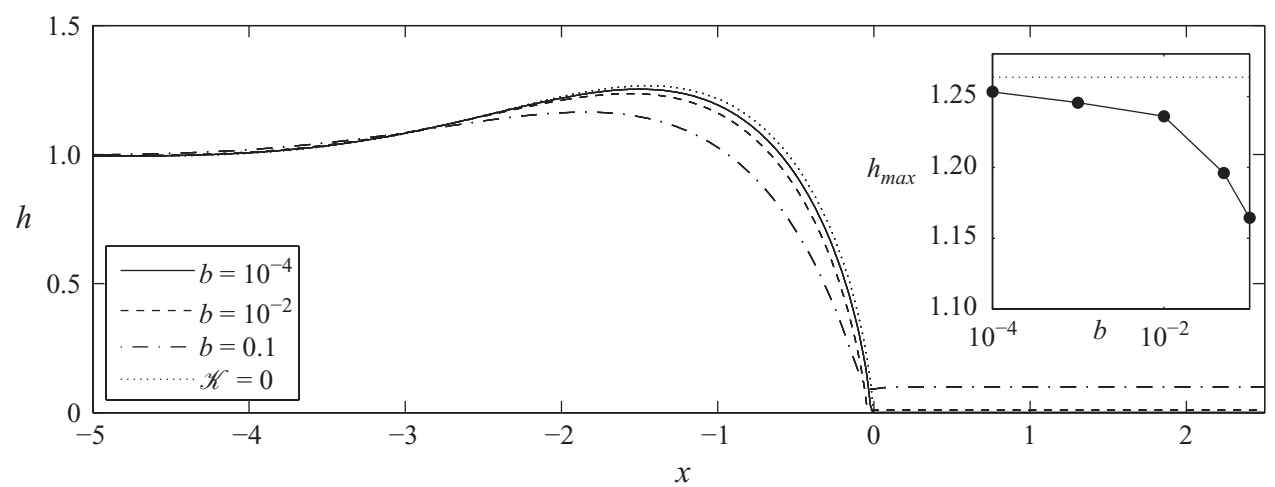

FIGURE 3. Steady front profiles for different $b$ together with the $\mathscr{K}=0$ solution. The inset shows the maximal crack width versus $b$.

\subsubsection{Steady planar fronts}

The steady planar front solutions are given by $[H(x, t), P(x, t)] \rightarrow\left[H_{f}(\xi), P_{f}(\xi)\right]$, where $\xi=x-c t$ and $c$ is the front speed. The front profile satisfies the integrodifferential system:

$$
c-1-c H_{f}=H_{f}^{3}\left(\frac{\mathrm{d} P_{f}}{\mathrm{~d} \xi}-1\right), \quad P_{f}(\xi)=\frac{1}{\pi} f_{-\infty}^{+\infty} \frac{\mathrm{d} H_{f}(\chi)}{\mathrm{d} \chi} \frac{\mathrm{d} \chi}{(\xi-\chi)} .
$$

Because $H_{f} \rightarrow b$ for $\xi \rightarrow \infty$, we find that the front speed $c=1+b+b^{2}$. In practice, although we could attack this system directly, we extract the front profiles from the end states of initial-value problems, ensuring convergence by moving into the frame translating with the front and computing for as long as necessary. We do this primarily because of the way in which we detect transverse instabilities, as described in $\S 2.2 .2$.

Examples of the steady fronts for differing values of $b$ are shown in figure 3 . A notable difference from the analogous surface tension problem (Spaid \& Homsy 1996) is that the solutions converge to a common limit as $b \rightarrow 0$, with the maximum width approaching a value just above 1.25. We interpret this limit as a solution to the zero-toughness fracture problem; indeed figure 3 also shows a recomputation of the $\mathscr{K}=0$ solution of Lister $(1990 a)$. 


\subsubsection{Transverse instability}

We explore the transverse stability of the planar states by introducing three-dimensional perturbations of the form $h=H(x, t)+\eta(x, t) \exp (\mathrm{i} k y)$ and $p=P(x, t)+\varpi(x, t) \exp (\mathrm{i} k y)$, and linearizing in the amplitudes $\eta(x, t)$ and $\varpi(x, t)$, where $k$ is the transverse wavenumber. The amplitudes satisfy the linear equations

$$
\eta_{t}=\partial_{x}\left[3 H^{2} \eta\left(P_{x}-1\right)+H^{3} \varpi_{x}\right]-k^{2} H^{3} \varpi
$$

and

$$
\varpi(x, t)=-\frac{k}{\pi} \int_{-\infty}^{\infty} \eta(z, t) \frac{\mathrm{K}_{1}(k|x-z|)}{|x-z|} \mathrm{d} z
$$

subject to $\eta \rightarrow 0$ as $x \rightarrow \pm \infty$. The integral in (2.16) involves the modified Bessel function $\mathrm{K}_{1}(x)$ (Abramowitz \& Stegun 1969) and follows from (2.8) on using the Fourier cosine transform

$$
\int_{0}^{\infty} \frac{\cos k y}{\left(y^{2}+x^{2}\right)^{3 / 2}} \mathrm{~d} y=\frac{k}{|x|} \mathrm{K}_{1}(k|x|) .
$$

We solve these equations by again transforming into the frame moving with the front, and then searching for normal mode solutions with time dependence $\exp (\lambda t)$ and growth rate $\lambda(k)$. We use the front solutions extracted from the initial-value computations and the same treatment of spatial derivatives and the integral to convert (2.15) into a matrix eigenvalue problem. Sample growth rates, $\lambda(k)$, computed in this fashion are shown in figure 4 , and reveal a band of unstable modes at sufficiently small values of $k$.

The growth rates shown in figure $4(a)$ have a maximum near $k=0.5$ and a higher wavenumber cutoff near $k=0.8$ for $b \rightarrow 0$. The most unstable eigenfunction $\eta$ is strongly localized to the fluid front, indicating that the main effect is to distort that front into an array of transverse fingers. The physical mechanism for the instability is similar to that for a capillary ridge, discussed in Spaid \& Homsy (1996): if one imagines rearranging the fluid to create alternating thick and thin regions along the fluid front in the transverse direction, then the thicker (thinner) portions have lower (higher) viscous drag and propagate faster (slower). The thickened fingers subsequently draw in more fluid from the retarded thinned regions to either side, which continues to reduce the drag and further accelerates the fingers. Spaid \& Homsy (1996) employed an energy analysis to unequivocally identify this mechanism for the advancing contact line; the same methodology can be adapted to the current problem.

\subsubsection{Long transverse waves}

Analytical results follow in the limit of long transverse wavelength, $k \ll 1$. In this limit, we introduce the asymptotic sequences $\lambda=k^{2} \lambda_{2}+\cdots$ and

$$
\eta(\xi)=\frac{\mathrm{d} H_{f}}{\mathrm{~d} \xi}+k^{2} \eta_{2}(\xi)+\cdots, \quad \varpi(\xi)=\frac{\mathrm{d} P_{f}}{\mathrm{~d} \xi}+\frac{(1-b)}{2 \pi} k^{2} \log k+k^{2} \varpi_{2}(\xi)+\cdots .
$$

The final relation is guided by the small-argument expansion of the modified Bessel function $\mathrm{K}_{1}(z)$ (Abramowitz \& Stegun 1969), which determines the $O\left(k^{2} \log k\right)$ correction and implies that

$$
\int_{-\infty}^{\infty} \frac{\mathrm{d} H_{f}(\chi)}{\mathrm{d} \chi} \frac{\mathrm{K}_{1}(k|\xi-\chi|)}{|\xi-\chi|} \mathrm{d} \chi=\frac{\mathrm{d} P_{f}}{\mathrm{~d} \xi}+o\left(k^{2}\right)
$$


(a)

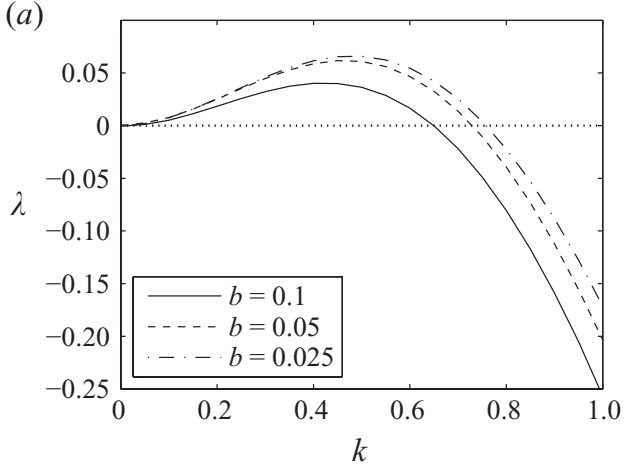

(b)

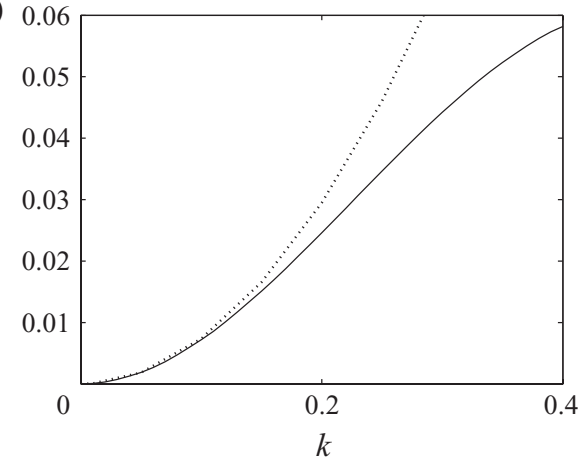

(c)

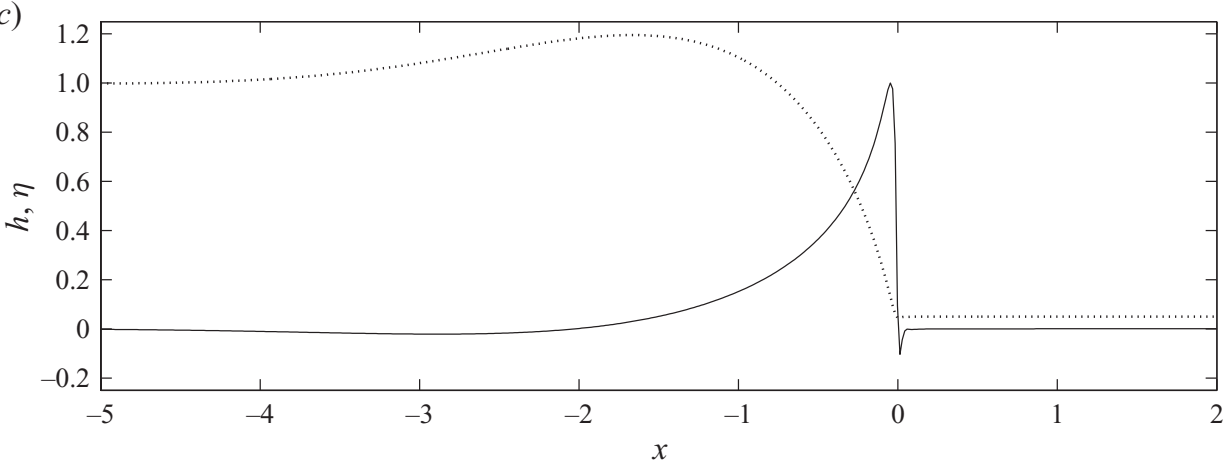

FiguRE 4. (a) Growth rates $\lambda$ versus $k$ for $b=0.1,0.05$ and 0.025. (b) A magnification at small $k$, comparing numerical results (solid) with the long-wave approximation in (2.21) (dotted) for $b=0.05$. In (c), the eigenfunction for the maximal growth rate is shown (solid) along with the front profile (dotted) also for $b=0.05$.

given that $\mathrm{d} H_{f} / \mathrm{d} \xi \sim O\left(\xi^{-3}\right.$ ), as $\xi \rightarrow \pm \infty$ (which follows from generalizing results presented by Spence et al. 1987).

Introducing these long-wave forms into the perturbation equation (2.15) provides, at order $k^{2}$,

$$
\lambda_{2} \frac{\mathrm{d} H_{f}}{\mathrm{~d} \xi}-c \frac{\mathrm{d} \eta_{2}}{\mathrm{~d} \xi}=\frac{\mathrm{d}}{\mathrm{d} \xi}\left[3 H_{f}^{2} \eta_{2}\left(\frac{\mathrm{d} P_{f}}{\mathrm{~d} \xi}-1\right)+H_{f}^{3} \frac{\mathrm{d} \varpi_{2}}{\mathrm{~d} \xi}\right]-H_{f}^{3} \frac{\mathrm{d} P_{f}}{\mathrm{~d} \xi} .
$$

Integrating this equation in $\xi$ from $-\infty$ to $+\infty$, and imposing the boundary conditions $\eta_{2} \rightarrow 0$ and $\varpi_{2} \rightarrow 0$ as $\xi \rightarrow \pm \infty$, leads to

$$
\lambda \sim \lambda_{2} k^{2}=\frac{k^{2}}{1-b} \int_{-\infty}^{\infty}\left(1+b+H_{f}\right)\left(H_{f}-1\right)\left(H_{f}-b\right) \mathrm{d} \xi .
$$

This prediction is also included in figure $4(b)$ and confirms the fidelity of the numerical calculation.

\subsection{Finite-volume releases}

\subsubsection{Initial-value problems}

A numerical solution to the planar initial-value problem (2.12)-(2.13) with (2.10) is shown in figure 5 . The top-hat-like initial inflation collapses quickly along the conduit under buoyant acceleration. A sharp fluid front develops, which eventually advances algebraically in time, spanning a narrowing section of the conduit and waning in 
(a)

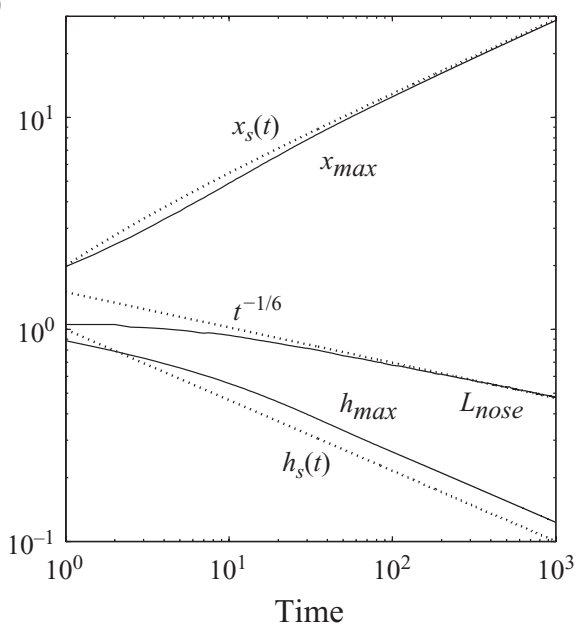

(e)

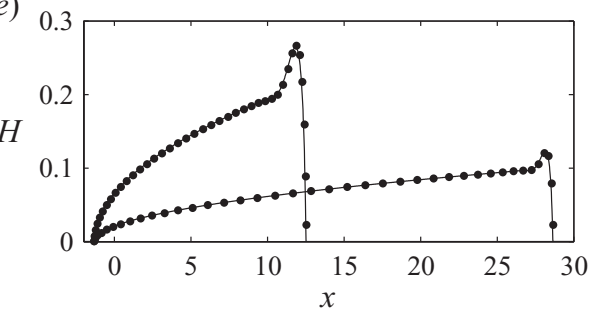

(b)

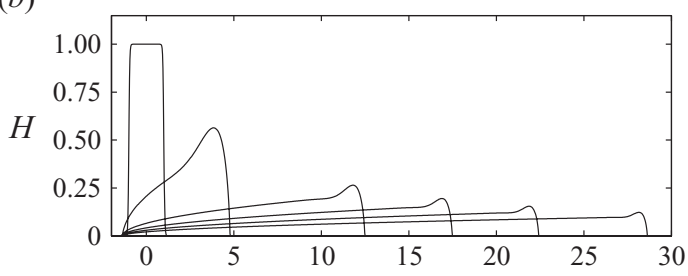

(c)

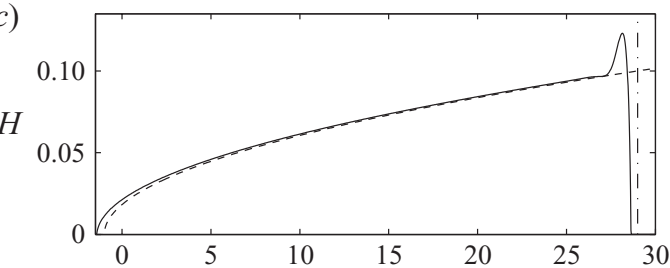

(d)

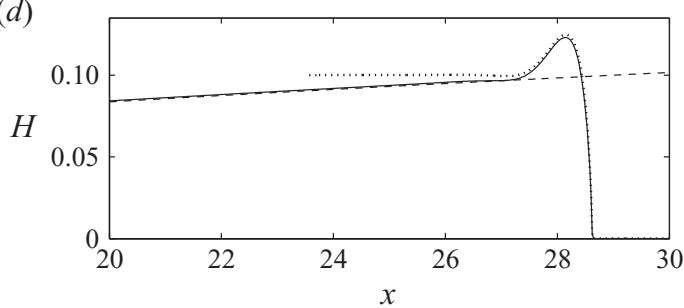

FIGURE 5. The evolution of the finite volume crack starting from (2.10) with $b=10^{-4}$. In $(a)$ the temporal evolution of the maximal half-thickness, $h_{\max }$, the position where $H=2 b$, denoted by $x_{\max }$, and $L_{\text {nose }}$, the length between $x_{\max }$ and the maximum opening are shown. The dotted lines indicate the predictions of $\S 2.3 .2$. In $(b)$, snapshots of $H(x, t)$ at $t=0,10,100,250,500$ and 1000 are shown. In $(c)$, a snapshot of $H(x, t)$ at $t=1000$ (solid) is shown along with the corresponding slot solution (2.22) (dashed) and the position $x_{s}$ in (2.23) (vertical dot-dashed). In addition, $(d)$ at $t=1000$ shows the front region in more detail, with the dashed line again showing (2.22) and the dotted line showing the rescaled steadily propagating front solution. In (e), we show the profile of the $b=10^{-4}$ solution at $t=100,1000$ (solid), together with those of a $\mathscr{K}=0$ computation (dots).

strength. More specifically, the tip of the front, defined as the position $x_{\max }$, where $H=2 b$, converges to $x_{\max }=O\left(t^{1 / 3}\right)$; the width of the front, as measured by the length from the tip of the front to the maximum opening $L_{n o s e}=O\left(t^{-1 / 6}\right)$; and the maximum opening $\max (h)=h_{\max }(t)=O\left(t^{-1 / 3}\right)$. The front leaves behind a gradually thinning and tapered slot whose thickness decreases as $O\left(t^{-1 / 2}\right)$. These algebraic dependences can all be extracted by a scaling analysis of the equations, and reflect an underlying matched asymptotic solution that takes a self-similar form in the two distinct regions (the front and the tapered slot; cf. Roper \& Lister 2007).

Again, the solutions for small $b$ approach a common limit corresponding to a zero-toughness solution with a genuine fracture edge. This is shown in figure 5(e), which compares a solution with $b=10^{-4}$ to one with $\mathscr{K}=0$. The latter computation explicitly calculates the fracture edge positions $X_{1}(t)$ and $X_{2}(t)$ by using a fixed grid on an expanding domain $0 \leqslant \tilde{x} \leqslant 1$, defined by $\tilde{x}=\left[x-X_{1}(t)\right] /\left[X_{2}(t)-X_{1}(t)\right]$, and determining ordinary differential equations for $X_{1}(t)$ and $X_{2}(t)$ by requiring that $h_{t}=0$ at those edges (Balmforth et al. 2006). The resulting partial differential equations are then spatially discretized (it is advantageous to cluster gridpoints at the ends to 

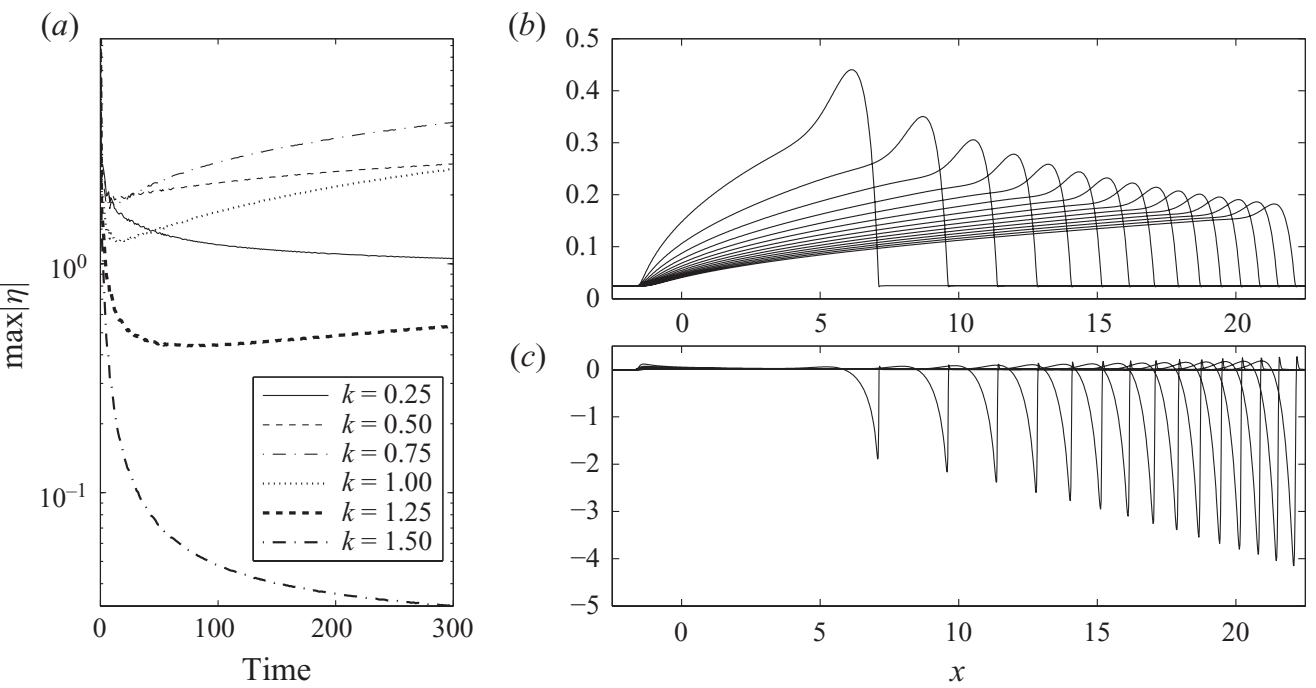

(c)

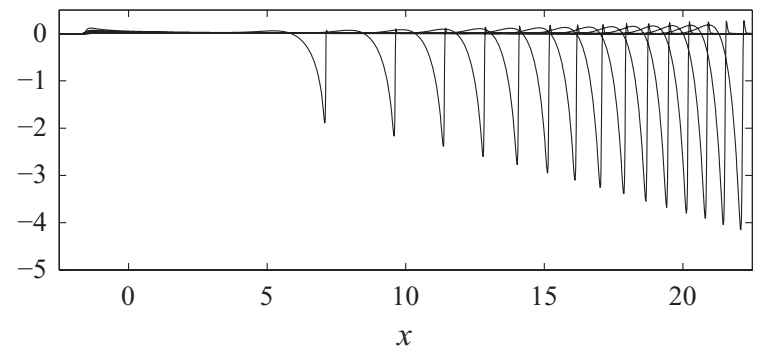

FIGURE 6. Solution of the initial-value problem for non-planar perturbations to constant-volume releases with $b=0.025$. (a) The evolution of $\max (|\eta|)$ versus time for $k=0.25,0.5,0.75,1,1.25,1.5 .(b, c)$ Snapshots of the base state $h(x, t)$ and the perturbation $\eta(x, t)$, for $k=0.75$, at times $t=20,40, \ldots, 300$.

accurately capture the sharp gradients; a non-uniform Chebyshev-Lobatto grid with 400 points is used) and integrated in time.

As for the case of constant flux, the planar fronts that emerge in figure 5 develop a pronounced ridge, and for related physical reasons, this structure may again suffer non-planar fingering instabilities. Unlike the steadily propagating constant-flux fronts, however, the constant-volume releases lead to structures that slow down and decrease in strength as they collapse, due to the waning flux feeding them. It is unclear whether the linear fingering instability survives this depletion of the basic state. On the other hand, the front sharpens as it wanes, to provide a partly compensating effect.

To determine the fate of the fingering instability, we compute solutions to the perturbation equations (2.15) and (2.16) in tandem with the planar front equations (2.12)-(2.13), beginning from the initial conditions $\eta(x, 0)=h_{x}(x, y, 0)$ and (2.10). A sample solution for $k=0.75$ is shown in figure 6 . In this example, the pre-cut conduit has a half-thickness of $b=0.025$, which is not particularly small, but needed in order to resolve the perturbation amplitudes, which develop severe peaks in the vicinity of the front. The main consequence of this choice of $b$ is that the ridge at the front is slightly shallower than it would be for $b \rightarrow 0$, reducing the potential for a fingering instability. Nevertheless, the solution clearly displays an amplification in the perturbation amplitude, defined as $\max (|\eta|)$.

Figure 6 also displays results for several other values of transverse wavenumber, $k$. For the duration of the computation, the modes with the lowest and highest wavenumbers $(k=0.25$ and 1.5$)$ display no appreciable growth, whilst the intermediate wavenumbers all become amplified to a degree depending on $k$. This suggests a $k$-dependent amplification with a form reminiscent of the growth rates for constantflux fronts shown in figure 4. However, figure 6 also demonstrates that growth is no longer exponential in time. We offer some rationale for the observed behaviour in $\S 2.3 .3$. 


\subsubsection{Self-similar planar fronts}

For the tapered slot behind the front, following Spence \& Turcotte (1990), we first note that when the elastic pressure term becomes small (and which we show later is relevant over this region), (2.12) reduces to $H_{t} \approx-3 H^{2} H_{x}$. Although this equation can be solved in general by the method of characteristics, the form of the initial condition dictates that the solution takes a self-similar form $H=F(Z)$, where $Z=(x+1) / t$, with a shock at its leading edge (bearing in mind that the trailing edge $x=-1$ barely moves). Demanding that the function $F(Z)$ solve the governing equation indicates that

$$
H \approx \sqrt{\frac{x+1}{3 t}},
$$

which is included in figure $5(\mathrm{c})$. Moreover, because most of the fluid is contained in the slot, mass conservation determines the location $x=x_{s}(t)$ of the shock at the leading edge, plus the maximum opening $h\left(x_{s} ; t\right)$, for $b \ll 1$,

$$
x_{s}(t)=-1+3 t^{1 / 3}
$$

and

$$
h\left(x_{s}, t\right)=h_{s}(t)=t^{-1 / 3},
$$

which are compared with the numerical results in figure 5 .

In the vicinity of the front, we introduce the time-dependent coordinate transformation,

$$
x=-1+3 t^{1 / 3}+t^{-1 / 6} X,
$$

to capture the overall advance of the front together with its shrinking width. We then search for a self-similar solution of the form

$$
H(x, t)=t^{-1 / 3} f(X) \quad \text { and } \quad P(x, t)=t^{-1 / 6} \Phi(X) .
$$

The scaling of the pressure follows from (2.13):

$$
P(x, t) \rightarrow \frac{1}{\pi t} f_{S} \frac{F_{Z}(Z) \mathrm{d} Z}{(x+1-t Z)}+\frac{1}{\pi t^{1 / 3}} f_{F} \frac{f_{X}(X) \mathrm{d} X}{\left(x+1-3 t^{1 / 3}-t^{-1 / 6} X\right)},
$$

where the integral is divided into contributions from the slot $(S)$ and the front $(F)$. When $x=t Z-1$ lies inside the slot, the first integral dominates, implying $P \sim t^{-1}$, and therefore $P_{x} \sim O\left(t^{-2}\right)$, justifying the neglect of the pressure gradient over this region. If $x$ lies inside the front region, on the other hand, we make the transformation in (2.25), discovering that the second term dominates and is $O\left(t^{-1 / 6}\right)$ :

$$
P(x, t)=t^{-1 / 6} \Phi(X) \sim \frac{1}{\pi t^{1 / 6}} f_{-\infty}^{\infty} \frac{\mathrm{d} f(\chi)}{\mathrm{d} \chi} \frac{\mathrm{d} \chi}{(X-\chi)} .
$$

Equation (2.12) can now be reduced at leading order to

$$
-f_{X} \sim\left[f^{3}\left(\Phi_{X}-1\right)\right]_{X} .
$$

However, (2.28) and (2.29) are identical to the system satisfied by our steadily propagating, constant-flux fronts for $b \ll 1$, with the travelling-wave coordinate $\xi$ replaced by $X$. Thus, $f(X) \equiv H_{f}(X)$. We illustrate how the rescaled solution within the front region collapses to the constant-flux front in figure $5(d)$; the rescaled solution is positioned using the computed front location. 


\subsubsection{Self-similar instabilities}

The similarity analysis can also be adapted to the linear-stability problem. Using the same rescalings as above, we search for perturbation solutions that are exponentially localized to the front region. A key detail of these self-similar perturbations is that in addition to rescaling $x=-1+3 t^{1 / 3}+t^{-1 / 6} X$, we also rescale the transverse coordinate and therefore the corresponding wavenumber:

$$
Y=y t^{1 / 6} \text { and } \quad \kappa=k t^{-1 / 6} .
$$

Thus, we set

$$
\eta(x, t) \mathrm{e}^{\mathrm{i} k y}=\zeta(X, t) \mathrm{e}^{\mathrm{i} \kappa Y} \quad \text { and } \quad \varpi(x, t) \mathrm{e}^{\mathrm{i} k y}=t^{1 / 6} \phi(X, t) \mathrm{e}^{\mathrm{i} \kappa Y},
$$

where the scaling of $\varpi(x, t)$ is guided by the development of (2.16), which in view of the localization of the eigenfunction to the front provides an analogue of (2.27):

$$
\varpi(x, t) \rightarrow t^{1 / 6} \phi(X, t) \sim-t^{1 / 6} \frac{\kappa}{\pi} \int_{-\infty}^{\infty} \zeta(\chi, t) \mathrm{K}_{1}(\kappa|X-\chi|) \frac{\mathrm{d} \chi}{|X-\chi|} .
$$

Note that this pressure perturbation decays exponentially beyond the front region, justifying the search for localized eigenfunctions in $\zeta(X, t)$.

Equation (2.15) now reduces to

$$
t^{1 / 2} \zeta_{t}-\zeta_{X} \sim \frac{\partial}{\partial X}\left[3 f^{2} \zeta\left(\Phi_{X}-1\right)+f^{3} \phi_{X}\right]-\kappa^{2} f^{3} \phi,
$$

where terms $O\left(t^{-1 / 2}\right)$ have been omitted. If we set

$$
\zeta(X, t)=\hat{\zeta}(X) \mathrm{e}^{\lambda(\kappa) \tau}, \quad \text { with } \quad \tau=2 t^{1 / 2}
$$

then (2.32) and (2.33) recover the linear eigenvalue problem for the steadily propagating constant-flux fronts. In other words, the computations of figure 4 can be translated into predictions for the growth rate $\lambda(\kappa)$ for self-similar instabilities. Notably, the modes are expected to amplify exponentially in $\tau \propto \sqrt{t}$, but with the time-dependent rate $\lambda\left(k t^{-1 / 6}\right)$. That is, the effective growth rate sweeps across the dispersion curves of figure 4(a) from right to left as time advances (assuming that the thickness of the pre-cut conduit plays no role). Over times for which the effective wavenumber $\kappa \sim 1 / 2$ we sweep across the maximum of the curve of $\lambda(\kappa)$, the effective growth rate changes little, and we expect the instabilities to amplify exponentially in $\tau=2 \sqrt{t}$. On the other hand, over later times, when the self-similar wavenumber $\kappa$ has entered the long-wave regime where $\lambda(\kappa) \sim \lambda_{2} t^{-1 / 3} k^{2}$, we anticipate exponential growth in $t^{1 / 6}$. The computations, shown in figure 6 , broadly show this kind of behaviour in time (with the logarithm of the maximum perturbation amplitude varying like $t^{\alpha}$ with $1 / 6<\alpha<1 / 2)$. Moreover, perturbations with smaller values of $k$ begin to amplify at earlier times and then trail off sooner in comparison to the higher wavenumbers (the case with $k=1.5$ has apparently yet to enter the phase of evolution for which the perturbation is expected to amplify, which is not surprising in view of the fact that the effective wavenumber $k t^{-1 / 6} \approx 0.6$ has barely entered the unstable band by the end of the computation).

\section{Experiments}

\subsection{Set-up}

For the experiments, we fracture blocks of gelatin by the downward propagation of a crack filled with pure Rogers Golden Syrup, dyed with a small amount of food colouring to enhance visualization. The blocks were prepared by dissolving 
Tank: Length $59.6 \mathrm{~cm}$

Depth $29.7 \mathrm{~cm}$

Width $7.2 \mathrm{~cm}$

\begin{tabular}{|c|c|c|c|}
\hline $\begin{array}{l}\text { Golden syrup } \\
\text { Fluid density } \\
\text { Fluid viscosity }\end{array}$ & $\begin{array}{l}1421 \mathrm{~kg} \mathrm{~m}^{-3} \\
183 \mathrm{~Pa} \mathrm{~s}\end{array}$ & $\begin{array}{l}\text { Gelatin } \\
\text { Solid density } \\
\text { Poisson ratio } \\
\text { Shear modulus } \\
\text { Fracture toughness }\end{array}$ & $\begin{array}{l}1050 \mathrm{~kg} \mathrm{~m}^{-3} \\
0.5 \\
2913 \mathrm{~Pa} \\
89 \mathrm{~Pa} \mathrm{~m}^{1 / 2}\end{array}$ \\
\hline $\begin{array}{l}\text { Magma } \\
\text { Fluid density } \\
\text { Fluid viscosity }\end{array}$ & $\begin{array}{l}2600 \mathrm{~kg} \mathrm{~m}^{-3} \\
100 \mathrm{~Pa} \mathrm{~s}\end{array}$ & $\begin{array}{l}\text { Rock } \\
\text { Solid density } \\
\text { Poisson ratio } \\
\text { Shear modulus } \\
\text { Fracture toughness }\end{array}$ & $\begin{array}{l}2900 \mathrm{~kg} \mathrm{~m}^{-3} \\
0.25 \\
2 \times 10^{10} \mathrm{~Pa} \\
10^{6} \mathrm{~Pa} \mathrm{~m}^{1 / 2}\end{array}$ \\
\hline
\end{tabular}

TABLE 1. Experimental data. The Young's modulus for gelatin $E=8740 \mathrm{~Pa}$ was found from a classical compression test (allowing a small mass, $200.00 \times 10^{-3} \mathrm{~kg}$, to deform the surface vertically by $8 \times 10^{-3} \mathrm{~m}$, see Jaeger 1964 ; Oakenfull, Parker $\&$ Tanner 1988). As the material is almost incompressible, the Poisson ratio $v=1 / 2$, so the shear modulus is $G=E / 3$. The fracture toughness of the gelatin was estimated using an empirical relation suggested by Menand \& Tait (2002). Data for magma and rock are taken from Lister (1990b) and Rubin (1995); these imply a dimensionless fracture toughness of $\mathscr{K} \sim 5 \times 10^{-4}$.

$550 \mathrm{~g}$ of commercial gelatin powder (supplied by David Roberts Food Corporation, Mississauga, Ontario, Canada) in 111 of boiling water, and then leaving the solution to solidify in the tank. The top of the tank was covered to avoid evaporation while the gelatin set for $22 \mathrm{~h}$ in an air-conditioned room at $23^{\circ} \mathrm{C}$. Physical data for the experimental materials and the dimensions of the tank are summarized in table 1. Note that these values indicate that the Reynolds number of the flow inside the fingers is estimated to be $R e=\rho_{f} H V / \mu \sim 5.4 \times 10^{-5} \ll 1$, which certainly justifies the use of the Stokes approximation that underlies the lubrication model used for the fluid motion.

Once set, the gelatin blocks were prepared for the experiment by cutting slots of depth $4 \mathrm{~cm}$ at the top using a heated strip of metal with thickness $0.5 \mathrm{~mm}$. A given mass of viscous fluid was then quickly poured directly into the cuts to uniformly fill them over the length of the tank to a thickness of the order of a millimetre, as illustrated in figure 1(top). The fluid then drove a fracture into the gelatin underlying the pre-cut slots, and images of the shape of the ensuing cracks were recorded by a digital camera as those structures migrated towards the base of the tank.

\subsection{Observations and measurements}

As already illustrated in figure 1, the initially planar cracks lose their twodimensionality and develop fingers as predicted theoretically. Figure 7 displays a summary of measurements taken from the experiments. Figure $7(a)$ shows the number of 'fully developed' fingers versus the mass of fluid emplaced, along with a selection of images of the finger patterns; fingers were identified as fully developed when they had descended at least $2 \mathrm{~cm}$ below the initial planar crack, and provided they had a distinct minimum (ruling out occasional side branches). Figure $7(b, c)$ displays the average finger spacing and their width, again plotted against the emplaced fluid mass, with error bars showing the variations encountered amongst the multiple, differently shaped fingers. The number of fingers initially increases with the emplaced fluid mass, but eventually the dimensions of the box constrain the available space within which the fingers can evolve. Note that, although crack propagation was largely 


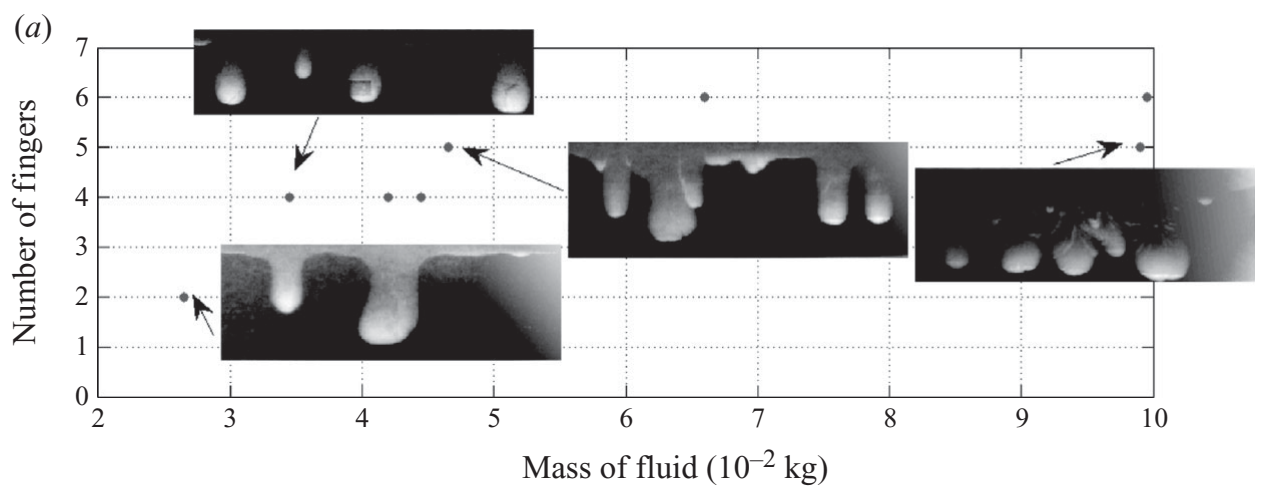

(b)

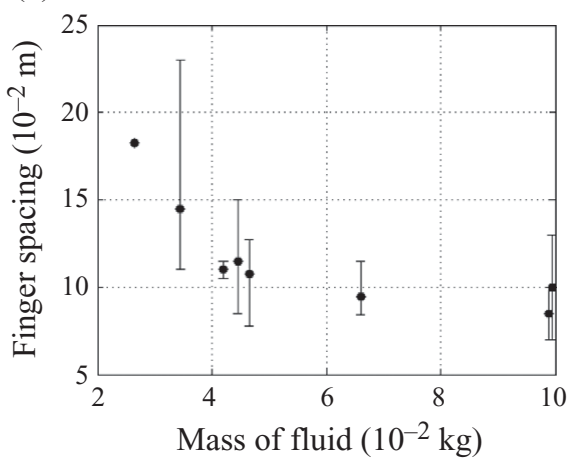

(c)

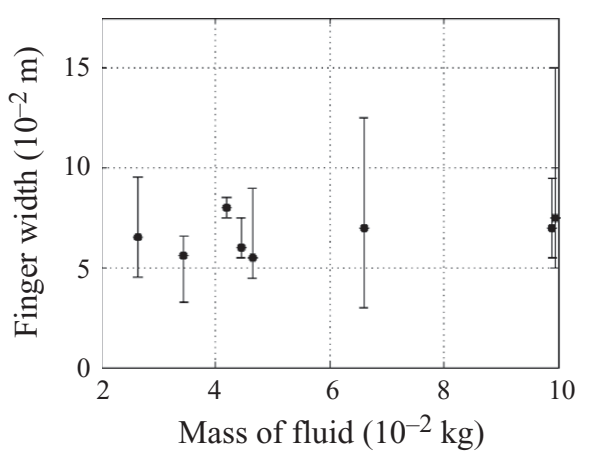

FIGURE 7. Experimental results for different emplaced masses of fluid. Shown in $(a)$ are the number of developed fingers; the inset photographs display four sample finger patterns. In $(b, c)$, the mean finger spacing and width are shown, respectively with error bars showing the variations amongst all the fingers appearing in the experiment.

in the vertical, a sideways deflection was impossible to eliminate, as illustrated by the reflections of the sides of the gelatin block in the angled mirrors bordering the tank in figure 1.

Multiple fingers developed in each experiment that was conducted. In some cases, as in figure 1, the initial instability appeared to set the finger spacing, which then persisted throughout the experiment. However, in other cases, the original finger pattern did not persist; a subset of the fingers grew at the expense of their neighbours, suppressing some of the fingers and increasing the overall spacing. Thus, nonlinear effects are clearly present and contribute to the finger development, and so linear dynamics cannot describe all the finger attributes.

More images and data from the experiment with emplaced mass $47.5 \mathrm{~g}$ are shown in figure 8. The sequence of photographs shows the development of two particular fingers. Figure 8(e) presents an image of the finger thickness based on the intensity of the green colour in the photograph at $t=912 \mathrm{~s}$; the intensity is approximately proportional to thickness (Liu, Paul \& Gollub 1993), and although the scale is arbitrary in the figure it is roughly equivalent to millimetres (the intensity scale can, in principle, be directly calibrated to thickness, but we did not attempt this refinement).

There are broadly three phases in the evolution evident in figure 8: an initial, planar phase; a period over which fingers appear and lengthen; and a final rundown phase in which finger growth is arrested. In the initial phase, the nearly planar crack 


$$
t=0 \mathrm{~s} \quad t=60 \mathrm{~s} \quad t=120 \mathrm{~s} \quad t=180 \mathrm{~s} \quad t=360 \mathrm{~s} \quad t=540 \mathrm{~s} \quad t=720 \mathrm{~s} \quad t=1140 \mathrm{~s} \quad t=1440 \mathrm{~s}
$$

(a)

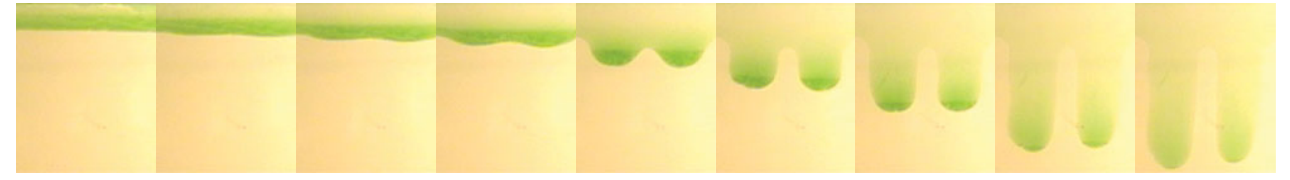

(b)

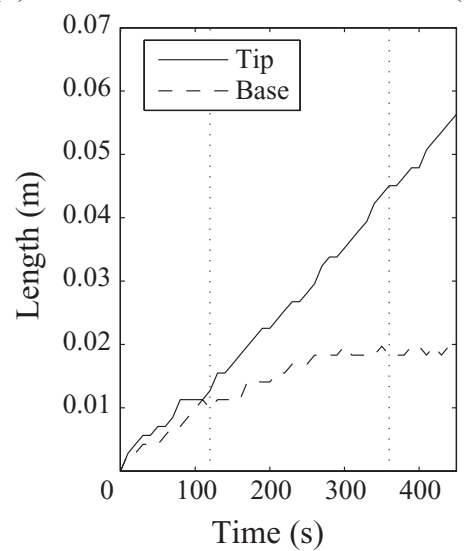

(c)

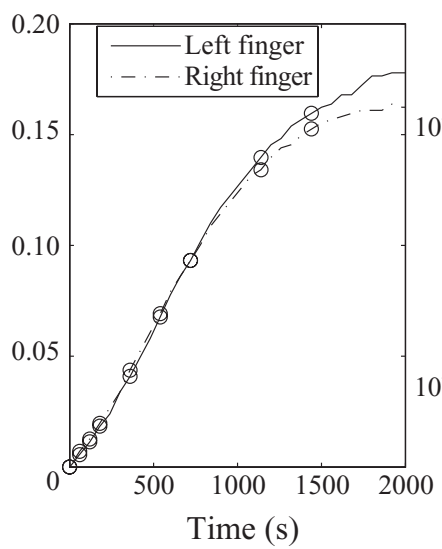

$(d)$

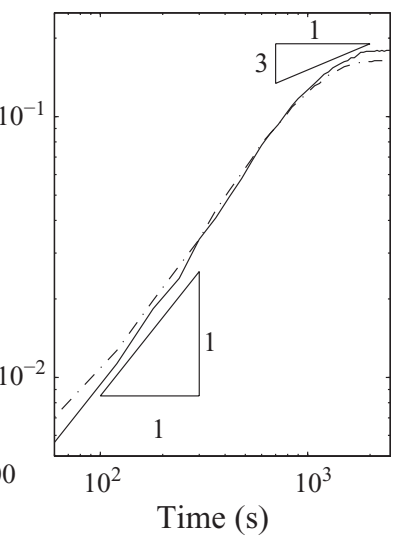

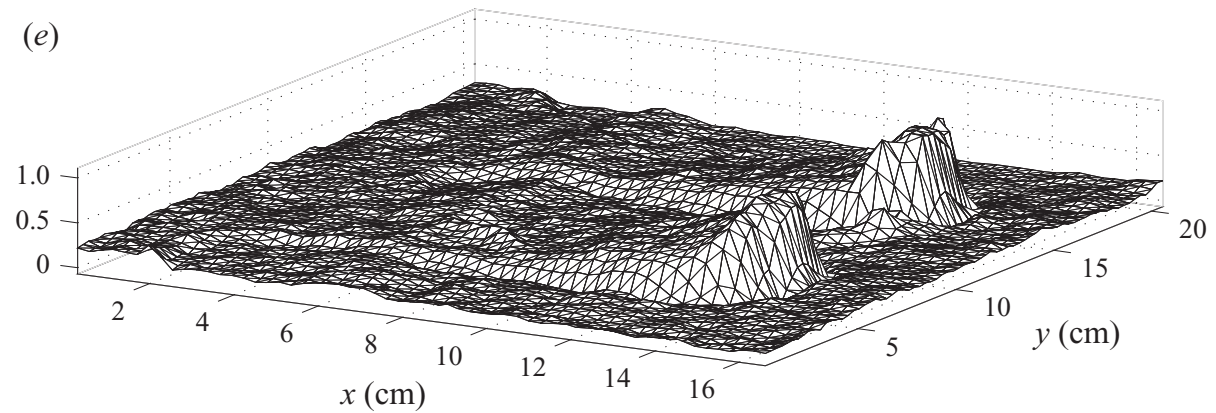

FIGURE 8. (Colour online) The time evolution of a pair of fingers for the experiment with $47.5 \mathrm{~g}$ of fluid). In $(a)$ a sequence of photographs is shown of the developing fingers at the times indicated; this pair corresponds to the rightmost pair in the photograph inset into the centre of figure $7(a)$. In $(b)$, a time series is presented of the positions of the finger tips and the base between them; the vertical dotted lines delineate the phases of evolution. In $(c)$ and $(d)$, the finger length, $L(t)$, is shown, first on a linear scale, and then logarithmically along with the scalings expected for the two-dimensional constant-volume theory (the circles indicate the times of the photographs shown in $(a))$. In $(e)$, the cross-sectional thickness of the fingers is displayed, extracted by measuring the intensity of the green colour in a photograph at $t=912 \mathrm{~s}$; as this intensity was not calibrated directly to actual thickness the scale is arbitrary.

descends approximately linearly in time, suggesting our method of triggering crack propagation is similar to supplying a constant flux. There is little distinction between the finger tips and bases between the fingers during this initial phase, which lasts for about $120 \mathrm{~s}$. The second phase of evolution then emerges as fingers appear and the tip and base positions diverge from one another; the bases slow down significantly, whereas the finger tips descend much as the original planar front. Eventually (at a time of about $360 \mathrm{~s}$ ) the final rundown sets in when the bases of the fingers come to a complete standstill because most of the fluid has drained into those conduits, 
trapping a finite volume within each finger. During the rundown, fingers continue to lengthen, slowing all the while; the $t^{1 / 3}$ scaling appropriate for two-dimensional propagation of a finite volume is shown for comparison in figure $8(d)$, but compares poorly with the data.

The fingers shown in figure 8 came to a standstill before they reached the base of the tank, in contrast to our constant-volume, zero-fracture-toughness solutions which slow down but never stop. Although the approach to the bottom surface might be partly responsible, fingers with a relatively small amount of trapped fluid often braked to a halt much higher in the tank, suggesting that some other effect is responsible for ending their downward progression (see also Taisne \& Tait 2009). One possibility is that the three-dimensional structure of the finger is responsible, although it is not clear how the geometry could affect the propagation in this fashion, especially given that one might expect the localization of the flow to drive the crack more effectively.

A second possibility is that the finite fracture toughness of the gelatin is able to arrest the propagation of a fingered crack driven by a finite volume of fluid. To gauge the merit of this proposal, we use dimensional analysis to determine that buoyancy becomes comparable to fracture toughness for a length of the order of $(K / \beta)^{2 / 3}$ (Taisne \& Tait 2009), which is equivalent to demanding that $\mathscr{K}$ in (2.11) is order one and then replacing $G \mathscr{H}$ using (2.4) to furnish an estimate of $\mathscr{L}$. We compare this distance with the length of that part of the crack containing most of the fluid; should $(K / \beta)^{2 / 3}$ exceed that length, we conclude that fracture toughness may control the dynamics. For our experiments, $(K / \beta)^{2 / 3} \sim 9 \mathrm{~cm}$, which is certainly of the order of or greater than the lengths of the fluid-filled cracks that came to a halt (see figure 8 ). Although this argument does not demonstrate that fingers halt because of the fracture toughness, it does suggest that the explanation may be plausible.

\section{Concluding remarks}

Fluid-filled and buoyancy-driven planar cracks develop bulges near the fracture tip that may suffer three-dimensional fingering instabilities by a similar mechanism that allows an advancing fluid contact line to finger. In this article, we have demonstrated both experimentally and theoretically that such instabilities do indeed occur, despite the fact that aspects of the fracture problem make the study more challenging than the corresponding surface tension problem.

Theoretically, the non-local dependence of the elastic forces on the crack shape leads us to an integro-differential problem to solve once we make the conventional lubrication approximation for the fluid and describe the solid by linear elasticity (Spence et al. 1987; Lister 1990a). To simplify this problem, and especially the linearstability analysis for three-dimensional perturbations about steady planar fronts, we avoided the introduction of a genuine fracture altogether, by considering fluid propagation along pre-cut, but narrow conduits. The precise technical issue is that linear perturbations are formally singular at the crack tip due to the shape of the underlying crack and the requirement that this tip is shifted by the perturbation. Nevertheless, we showed that as the thickness of the initial conduit approached zero, the solutions converged to true fracture solutions provided the elastic material had zero fracture toughness.

Although negligible fracture toughness may characterize geological applications, for our experiments the gelatin block actually possessed a non-negligible fracture toughness (according to the numbers quoted in table 1, the dimensionless fracture toughnesses, $\mathscr{K}$, are $O(1))$. Though this places those experiments in a different 
physical regime, the fact that we observed fingering also in the laboratory indicates that the instability does not rely on vanishing fracture toughness. Indeed, although our theoretical discussion took $\mathscr{K}=0$ in order to avoid the details of the crack edge, steadily propagating solutions with finite toughness are available (Lister 1990a) and our long-wave perturbation analysis $(\$ 2.2 .3)$ can be adapted to gauge stability in that limit. As in the surface tension problem, the primary feature driving instability is the extent of the fluid ridge near the crack tip, which increases with toughness, as shown by Lister $(1990 a)$. This suggests that we have actually explored the least unstable situation from the theoretical side.

The experiments themselves were particularly challenging: the gelatin required some care in its preparation in order to minimize problems with transparency and homogeneity, difficulties that were compounded by the need to have a relatively long tank in order to unambiguously observe multiple finger wavelengths. Also, we were then forced to use a tank that was not specially wide. Consequently, the crack is influenced by the proximity of the sidewalls, not least because the cracks often drifted horizontally, sometimes to intersect those walls and thereafter peel the gelatin away lower down. Fractures without simple geometry also sometimes emerged; in weaker gelatin, the crack developed complicated petal or bladed patterns. All of these complications are presumably why these instabilities have not been documented before.

The differing fracture toughness precludes us from comparing the theory with the experiment in detail. Nevertheless, it is worth pointing out that the characteristic length scales, based on the physical parameters given in table $1, \mathscr{H}$ and $\mathscr{L}$ are about $3 \mathrm{~mm}$ and $7 \mathrm{~cm}$, respectively, for the syrup-gelatin configuration. These scales are certainly representative of experimental crack thicknesses and finger widths and spacings. More appropriate is a comparison with the geological application. Unfortunately, physical data for magmatic processes are unreliable, but a suggestive set of values is given in table 1. These numbers (along with the flux estimate $Q=500 \mathrm{~m}^{3} \mathrm{~s}^{-1}$ ) suggest length scales of $\mathscr{H} \sim 3 \mathrm{~m}, \mathscr{L} \sim 6 \mathrm{~km}$, which are not unreasonable values of dyke extent. The most unstable wavelength for a constant-flux crack is then predicted to be $60 \mathrm{~km}$, which is satisfyingly within the $50-70 \mathrm{~km}$ spacings of volcanoes observed along the Mariana Arc (Bloomer et al. 1989).

T.T. thanks the Rhône-Alpes Region (France) for their generous financial support through the Explo'RA Sup grant. The authors thank NSERC (Canada) for support through the Discovery Grant Scheme. R.V.C. gratefully acknowledges many useful and interesting discussions with Steve Tait and Benoit Taisne (IPGP Paris).

\section{REFERENCES}

Abramowitz, M. \& Stegun, I. A. 1969 Handbook of Mathematical Tables. Dover.

Adachi, A., Siebrits, E., Peirce, A. \& Desroches, J. 2007 Computer simulation of hydraulic fractures. Intl J. Rock Mech. Mining Sci. 44, 739-757.

Balmforth, N. J., Craster, R. V., Rust, A. C. \& Sassi, R. 2006 Viscoplastic flow over an inclined surface. J. Non-Newtonian Fluid Mech. 139, 103-127.

Bloomer, S. H., Stern, R. J. \& Smoot, N. C. 1989 Physical volcanology of the submarine Mariana and Volcano arcs. Bull. Volcanol. 51, 210-224.

Boschi, C., Dini, A., Dallai, L., Ruggieri, G. \& Gianelli, G. 2009 Enhanced $\mathrm{CO}_{2}$-mineral sequestration by cyclic hydraulic fracturing and Si-rich fluid infiltration into serpentinites at Malentrata (Tuscany, Italy). Chem. Geol. 265, 209-226. 
Craster, R. V. \& Matar, O. K. 2009 Dynamics and stability of thin liquid films. Rev. Mod. Phys. 81, 1131-1198.

Desroches, J., Detournay, E., Lenoach, B., Papanastasiou, P., Pearson, J. R. A., Thiercelin, M. \& Cheng, A. 1994 The crack tip region in hydraulic fracturing. Proc. R. Soc. Lond. A 447, $39-48$.

Economides, M. \& Nolte, K. (Eds.) 2000 Reservoir Stimulation, 3rd. edn. John Wiley \& Sons.

Freund, L. B. 1990 Dynamic Fracture Mechanics. Cambridge University Press.

Heimpel, M. \& Olson, P. 1994 Buoyancy-driven fracture and magma transport through the lithosphere: models and experiments. In Magmatic Systems (ed. M. P. Ryan), pp. 223-240. Academic.

HupPert, H. E. 1982 Flow and instability of a viscous current down a slope. Nature 300, 427-429.

IOAKIMIDIS, N. I. 1982 Application of finite-part integrals to the singular integral equations of crack problems in plane and three-dimensional elasticity. Acta Mechanica 45, 31-47.

JAEGER, J. C. 1964 Elasticity, Fracture and Flow, 2nd edn. Methuen.

Krawczynski, M. J., Behn, M. D., Das, S. B. \& Joughin, I. 2009 Constraints on the lake volume required for hydro-fracture through ice sheets. Geophys. Res. Lett. 36, L10501.

ListeR, J. R. 1990a Buoyancy-driven fluid fracture: the effects of material toughness and of lowviscosity precursors. J. Fluid Mech. 210, 263-280.

ListeR, J. R. 1990 b Buoyancy-driven fracture: similarity solutions for the horizontal and vertical propagation of fluid-filled cracks. J. Fluid Mech. 217, 213-239.

Liu, J., Paul, J. D. \& Gollub, J. P. 1993 Measurements of the primary instabilities of film flows. J. Fluid Mech. 250, 69-101.

Menand, T. \& TaIt, S. 2001 A phenomenological model for precursor volcanic eruptions. Nature 411, 678-680.

Menand, T. \& TaIT, S. 2002 The propagation of a buoyant liquid-filled fissure from a source under constant pressure. J. Geophys. Res. B 107, 2306-2320.

Oakenfull, D. G., Parker, N. S. \& Tanner, R. I. 1988 Method for determining absolute shear modulus of gels from compression tests. J. Texture Stud. 19(4), 407-417.

Oron, A., Davis, S. H. \& Bankoff, S. G. 1997 Long-scale evolution of thin liquid films. Rev. Mod. Phys. 69, 931-980.

Roper, S. M. \& LisTeR, J. R. 2007 Buoyancy-driven crack propagation: the limit of large fracture toughness. J. Fluid Mech. 580, 359-380.

Rubin, A. M. 1995 Propagation of magma-filled cracks. Annu. Rev. Earth Planet Sci. 23, 287-336.

Silvi, N. \& Dussan V., E. B. 1985 On the rewetting of an inclined solid-surface by a liquid. Phys. Fluids 28, 5-7.

Spaid, M. A. \& Homsy, G. M. 1996 Stability of Newtonian and viscoelasticity dynamic contact lines. Phys. Fluids 8, 460-478.

Spence, D. A., Sharp, P. W. \& Turcotte, D. L. 1987 Buoyancy-driven crack propagation: a mechanism for magma migration. J. Fluid Mech. 174, 135-153.

Spence, D. A. \& TurcotTe, D. L. 1990 Buoyancy-driven magma fracture: a mechanism for ascent through the lithosphere and the emplacement of diamonds. J. Geophys. Res. 95 (B4), 51335139.

Taisne, B. \& Jaupart, C. 2009 Dike propagation through layered rocks. J. Geophys. Res. Solid Earth 114, B09203.

TAISNE, B. \& TAIT, S. 2009 Eruption versus intrusion? Arrest of propagation of constant volume, buoyant, liquid-filled cracks in an elastic, brittle host. J. Geophys. Res. 114, B06202.

TAKADA, A. 1990 Experimental study on propagation of liquid-filled crack in gelatin: shape and velocity in hydrostatic stress condition. J. Geophys. Res. B 95, 8471-8481.

Troian, S. M., Herbolzheimer, E., Safran, S. A. \& Joanny, J. F. 1989 Fingering instabilities of driven spreading films. Europhys. Lett. 10, 25-30.

TSAI, V. C. \& Rice, J. R. 2010 A model for turbulent hydraulic fracture and application to crack propagation at Glacier beds. J. Geophys. Res. 115, F03007.

WeERTMAN, J. 1971 Theory of water-filled crevasses in glaciers applied to vertical magma transport beneath oceanic ridges. J. Geophys. Res. 76, 1171-1183.

WeISS, J. 2004 Subcritical crack propagation as a mechanism of crevasse formation and iceberg calving. J. Glaciol. 50, 109-115. 